\title{
Swift detection of all previously undetected blazars in a micro-wave flux-limited sample of WMAP foreground sources
}

\author{
P. Giommi ${ }^{1,2}$, M. Capalbi ${ }^{1}$, E. Cavazzuti ${ }^{1,2}$, S. Colafrancesco ${ }^{3}$, A. Cucchiara ${ }^{4}$, A. Falcone ${ }^{4}$, J. Kennea ${ }^{4}$, R. Nesci ${ }^{5}$, \\ M. Perri ${ }^{1}$, G. Tagliaferri ${ }^{6}$, A. Tramacere ${ }^{5}$, G. Tosti $^{7}$, A. J. Blustin ${ }^{8}$, G. Branduardi-Raymont ${ }^{8}$, D. N. Burrows ${ }^{4}$, \\ G. Chincarini ${ }^{6}$, A. J. Dean ${ }^{9}$, N. Gehrels ${ }^{10}$, H. Krimm ${ }^{10}$, F. Marshall ${ }^{10}$, A. M. Parsons ${ }^{10}$, and B. Zhang ${ }^{11}$ \\ ASI Science Data Center, ASDC c/o ESRIN, via G. Galilei 00044 Frascati, Italy \\ e-mail: paolo.giommi@asi.it \\ 2 Agenzia Spaziale Italiana, Unitá Osservazione dell'Universo, via le Liegi, 2600198 Roma, Italy \\ 3 INAF - Osservatorio Astronomico di Roma via Frascati 33, 00040 Monteporzio, Italy \\ ${ }^{4}$ Department of Astronomy and Astrophysics, Pennsylvania State University, USA \\ 5 Universitá di Roma "La Sapienza” Dipartimento di Fisica P.le A. Moro, 2 00185, Roma, Italy \\ 6 INAF - Osservatorio Astronomico di Brera via Bianchi 46, 23807 Merate, Italy \\ 7 Dipartimento di Fisica, Universitá di Perugia, via A. Pascoli, Perugia, Italy \\ 8 UCL, Mullard Space Science Laboratory, Holmbury St. Mary, Dorking, Surrey RH5 6NT, UK \\ 9 University of Southampton, Highfield, S017 1BJ Southampton, UK \\ 10 NASA/Goddard Space Flight Center, Greenbelt, Maryland 20771, USA \\ 11 Department of Physics, University of Nevada, Las Vegas, NV 89154-4002, USA
}

Received 6 September 2006 / Accepted 25 January 2007

\begin{abstract}
Almost the totality of the bright foreground sources in the WMAP Cosmic Microwave Background (CMB) maps are blazars, a class of sources that show usually also X-ray emission. However, 23 objects in a flux-limited sample of 140 blazars of the WMAP catalog (first year) were never reported before as X-ray sources. We present here the results of 41 Swift observations which led to the detection of all these 23 blazars in the $0.3-10 \mathrm{keV}$ band. We conclude that all micro-wave selected blazars are X-ray emitters and that the distribution of the micro-wave to X-ray spectral slope $\left(\alpha_{\mu x}\right)$ of LBL blazars is very narrow, confirming that the X-ray flux of most blazars is a very good estimator of their micro-wave emission. The X-ray spectral shape of all the objects that were observed long enough to allow spectral analysis is flat and consistent with inverse Compton emission within the commonly accepted view where the radiation from blazars is emitted in a Sychrotron-Inverse-Compton scenario. We predict that all blazars and most radio galaxies above the sensitivity limit of the WMAP and of the Planck CMB missions are X-ray sources detectable by the present generation of X-ray satellites. An hypothetical all-sky soft X-ray survey with sensitivity of approximately $10^{-15} \mathrm{erg} \mathrm{cm}^{-2} \mathrm{~s}^{-1}$ would be crucial to locate and remove over 100000 blazars from CMB temperature and polarization maps and therefore accurately clean the primordial CMB signal from the largest population of extragalactic foreground contaminants.
\end{abstract}

Key words. quasars: general - cosmology: cosmic microwave background - radiation mechanisms: non-thermal

\section{Introduction}

Blazars are a type of AGN whose nuclear emission is dominated by strong non-thermal radiation that extends across the entire electromagnetic spectrum, from radio frequencies to the most energetic $\gamma$-rays. The typical observational properties of blazars include irregular, rapid and often very large variability, apparent super-luminal motion, flat radio spectrum, and large and variable polarization at radio and optical frequencies. These unusual properties are thought to be due to electromagnetic radiation emitted in a relativistic jet viewed closely along the line of sight (Blandford \& Rees 1978; Urry \& Padovani 1995). There are two types of blazars: BL Lacertae objects (or BL Lacs) that show no or very weak emission lines in their optical spectrum and Flat Spectrum Radio Quasars (FSRQs) whose spectrum includes all the broad emission lines typically seen in radio quiet AGN.

Traditionally, this type of sources has been found either in radio surveys or as counterparts of X-ray sources. More recently a significant number of new blazars have also been discovered at optical frequencies (e.g., Collinge et al. 2005).

The broad-band electromagnetic spectrum of blazars is composed of a synchrotron low-energy component that peaks (in $\log (v f(v))-\log (v)$ representation) between the far infrared and the X-ray band, followed by an inverse Compton component that has its maximum in the hard X-ray band or at higher energies, depending on the location of the synchrotron peak, and extends into the $\gamma$-ray or even the TeV energy band. Blazars with the synchrotron peak located at infrared frequencies are usually called Low energy peaked Blazars or LBL, while objects where the synchrotron emission reaches the UV/X-ray band are called High energy peaked Blazars or HBL (Padovani \& Giommi 1995). HBLs are almost exclusively of the BL Lac type, whereas LBL sources are both FSRQs and BL Lac objects and make up the large majority of the blazar population (e.g. Padovani et al. 2003; Giommi et al. 2006a). 
The micro-wave band is particularly effective for the selection of blazars, as in this energy region the flat spectrum emission from these sources is not diluted by steep non-nuclear components that are often observed at $\mathrm{cm}$ wavelengths. However, until very recently no surveys had sufficient sensitivity, contrast, and resolution to distinguish faint foreground sources from the much stronger Cosmic Microwave Background. The Wilkinson Microwave Anisotropy Probe (Bennett et al. 2003a), which is dedicated to the accurate measurement of the primordial fluctuations of the CMB at small angular scales, is the first experiment to provide such a capability. A catalog of 208 bright foreground sources was produced by Bennett et al. (2003b) by using the data from the first year of WMAP data. Giommi \& Colafrancesco (2004) have shown that the large majority of these sources $(>85 \%)$ are blazars. These authors also showed that a large number of fainter, non-resolved blazars can contaminate the primordial CMB fluctuation spectrum in a non-negligible way.

Although blazars only represent a small minority of the entire AGN population, their strong emission at all wavelengths allow them to stand out in those energy bands where other types of AGN are "quiet". Some of these spectral windows are presently poorly explored but are rapidly becoming the subject of extensive studies by current and near future satellites like WMAP, Planck (Giommi \& Colafrancesco 2004), AGILE and GLAST (Giommi et al. 2006a).

The Swift Gamma-Ray-Burst (GRB) Explorer (Gehrels et al. 2004) is a multi-frequency, rapid response space observatory that was launched on November 20, 2004. To fulfill its purposes Swift carries three instruments on board: the Burst Alert Telescope (BAT, Barthelmy et al. 2005) sensitive in the 15-150 keV band, the X-Ray Telescope (XRT, Burrows et al. 2005) sensitive in the $0.2-10.0 \mathrm{keV}$ band, and the UV and Optical Telescope (UVOT, Roming et al. 2005). The Swift observation program is fully dedicated to the discovery and rapid follow up of GRBs. However, as these elusive sources explode at random times and their frequency of occurrence is subject to large statistical fluctuations, there are periods when Swift is not engaged with GRB observations and can be used for other scientific purposes. In this context we have started a program to observe and monitor samples of blazars selected to represent different aspects of this class of sources.

In this paper we report the observations of a sample of 23 blazars detected at micro-wave frequencies by the WMAP satellite (Bennett et al. 2003a) that had no previous $\mathrm{X}$-ray observations, and that were not detected in the Rosat all Sky Survey. The XRT instrument on board Swift is particularly suitable for this project as it provides great flexibility and sufficient sensitivity to detect and determine the spectral shape of sources as faint as $\approx 10^{-13} \mathrm{erg} \mathrm{cm}^{-2} \mathrm{~s}^{-1}$ in short exposures.

\section{Sample description}

The blazar content of the WMAP catalog of bright foreground sources (Bennett et al. 2003b) is described in Giommi \& Colafrancesco (2004) and Giommi et al. (2006a). Out of the 208 sources in the catalog, 164 are previously known blazars, 13 are radio galaxies, 5 are steep spectrum radio QSOs and 2 are starburst galaxies; 17 sources still remain unidentified. The only objects in the WMAP catalog identified with Galactic sources are 2 planetary nebulae, whereas 5 objects have no radio counterparts at 1.4 or $5 \mathrm{GHz}$ and are probably spurious. This sample of blazars is flux limited at micro-wave frequencies and includes objects with rich multi-frequency observational data, especially at radio, optical, and X-ray frequencies. About 35 objects are also included in the 3rd EGRET catalog of gamma-ray sources (Hartman et al. 1999; Giommi et al. 2006a).

Within this sample, 42 objects have never been reported as X-ray sources and have no counterpart in the ROSAT all sky X-ray survey (Voges et al. 1999). In the following, we consider the subset of $23 \mathrm{X}$-ray undetected WMAP sources identified with blazars that have micro-wave flux larger than $0.8 \mathrm{Jy}$ at $41 \mathrm{GHz}$. This sample includes less than $20 \%$ of all the 140 identified blazars in the WMAP catalog that satisfy the above flux limit. The list of sources is given in Table 1 where Col. 1 gives the source name, Col. 2 gives the source number in the WMAP catalog, Cols. 3 and 4 give the right ascension and declination for the J2000.0 equinox, Col. 5 gives the redshift (when available), Col. 6 gives the radio flux density at $5 \mathrm{GHz}$, Col. 7 the micro-wave flux density at $41 \mathrm{GHz}$ from the WMAP cata$\log$, Col. 8 gives amount of Galactic $N_{\mathrm{H}}$ estimated from $21 \mathrm{~cm}$ measurements (Dickey \& Lockman 1990), and Col. 9 gives the blazar type (FSRQ or BL Lac)

\section{Swift XRT observations}

Swift performed a total of 41 observations of the blazars listed in Table 1.

The XRT is usually operated in Auto State mode which automatically adjusts the readout mode of the CCD detector to the source brightness, in an attempt to avoid pile-up (see, for details of the XRT observing modes Burrows et al. 2005; Hill et al. 2004). Given the low count-rate of our blazars most of the data were collected using the most sensitive Photon Counting (PC) mode; Windowed Timing (WT) mode was used in some cases with very short exposures.

We reduced the XRT data using the XRTDAS software (v1.8.0) developed at the ASI Science Data Center (ASDC) and distributed within the HEAsoft 6.0 .5 package by the NASA High Energy Astrophysics Archive Research Center (HEASARC). We selected photons with grades in the range 0-12 and used default screening parameters to produce level 2 cleaned event files.

\subsection{Image analysis and $X$-ray fluxes}

$\mathrm{X}$-ray images were accumulated from level 2 (cleaned and calibrated) event files, accepting photons with energy between 0.3 and $10 \mathrm{keV}$. Source count-rates were estimated using the DETECT routine within the XIMAGE.V 4.2 package. Net counts were then converted into flux assuming a power law spectrum with photon index equal to 1.5 and setting the amount of photoelectric absorption $\left(N_{\mathrm{H}}\right)$ equal to the Galactic value along the line of sight (see Table 1).

Table 2 summarizes the results. Column 1 gives the blazar name, Col. 2 gives the observation date, Col. 3 the XRT net exposure in seconds, Col. 4 gives the count-rate in the $0.3-10 \mathrm{keV}$ energy range, Cols. 5 and 6 give the observed X-ray flux in the $0.5-2$ and $2-10 \mathrm{keV}$ bands respectively, and Col. 7 gives the micro-wave (94 GHZ) to X-ray (1 keV) slope defined as follows:

$\alpha_{\mu x}=-\frac{\log \left(f_{1 \mathrm{keV}} / f_{94 \mathrm{GHz}}\right)}{\log \left(v_{1 \mathrm{keV}} / v_{94 \mathrm{GHz}}\right)}=-\frac{\log \left(f_{1 \mathrm{keV}} / f_{94 \mathrm{GHz}}\right)}{6.41}$,

where $f_{1 \mathrm{keV}}$ is the de-absorbed flux at $1 \mathrm{keV}$, k-corrected assuming a power law spectral slope of 1.5 , and $f_{(94 \mathrm{GHz})}$ is the WMAP flux density at $94 \mathrm{GHz}$ when available, or an extrapolation to $94 \mathrm{GHz}$ of the $41 \mathrm{GHz}$ flux given in Table 1 
Table 1. The sample: WMAP blazars with $41 \mathrm{GHz}$ flux larger that $0.8 \mathrm{Jy}$ for which no previous X-ray detection was available.

\begin{tabular}{|c|c|c|c|c|c|c|c|c|}
\hline Blazar name & $\begin{array}{c}\text { WMAP } \\
\text { Catalog } \\
\text { number } \\
\text { (2) }\end{array}$ & $\begin{array}{c}\text { RA } \\
\text { J2000.0 }\end{array}$ & $\begin{array}{c}\text { Dec } \\
\text { J2000.0 }\end{array}$ & Redshift & $\begin{array}{l}\text { Radio flux } \\
5 \mathrm{GHz}, \mathrm{mJy}\end{array}$ & $\begin{array}{c}\text { Micro-wave } \\
\text { flux } \\
41 \mathrm{GHz}, \mathrm{Jy} \\
\text { (7) }\end{array}$ & $\begin{array}{c}N_{\mathrm{H}} \\
\times 10^{20} \\
\mathrm{~cm}^{-2} \\
(8)\end{array}$ & Blazar type \\
\hline DW $0202+31$ & 085 & 020504.8 & +321229.1 & 1.466 & 934 & 1.3 & 6.7 & FSRQ \\
\hline PKS $0215+015$ & 096 & 021748.7 & +014448.1 & 1.715 & 1011 & 1.6 & 3.3 & FSRQ \\
\hline PKS 0511-220 & 127 & $\begin{array}{lll}05 & 13 & 49.1\end{array}$ & -215916.1 & 1.296 & 865 & 1.4 & 2.7 & FSRQ \\
\hline S5 0633+73 & 087 & 063921.9 & +732457.0 & 1.85 & 711 & 1.1 & 7.0 & FSRQ \\
\hline 1Jy 0805-077 & 133 & 080815.4 & -075107.9 & 1.837 & 1599 & 1.4 & 9.8 & FSRQ \\
\hline 1Jy $1030+415$ & 103 & 103303.5 & +411605.1 & 1.117 & 439 & 1.5 & 1.2 & FSRQ \\
\hline S5 1044+71 & 083 & 104827.4 & +714333.9 & 1.15 & 1900 & 1.1 & 3.5 & FSRQ \\
\hline PKS 1206-238 & 172 & 120902.3 & -240619.0 & 1.299 & 1073 & $1.1^{*}$ & 7.4 & BL Lac \\
\hline 1Jy 1213-172 & 173 & 121546.7 & -173145.8 & - & 1744 & 1.6 & 4.3 & FSRQ \\
\hline PKS 1313-333 & 182 & 131607.8 & -333858.9 & 1.21 & 1093 & 1.7 & 4.8 & FSRQ \\
\hline 1Jy 1406-076 & 203 & 140856.4 & -075224.9 & 1.494 & 1080 & 1.5 & 2.8 & FSRQ \\
\hline 1Jy $1424-418$ & 191 & 142756.2 & -420620.8 & 1.522 & 2597 & 2.6 & 8.3 & FSRQ \\
\hline 1Jy $1548+056$ & 007 & 155035.0 & +052710.0 & 1.422 & 1766 & 1.8 & 4.3 & FSRQ \\
\hline PKS 1725-795 & 186 & 173340.7 & -793553.8 & - & 419 & 1.0 & 7.6 & FSRQ \\
\hline S2 1848+28 & 028 & 185027.4 & +282512.0 & 2.56 & 1013 & 0.9 & 12.2 & FSRQ \\
\hline 3C 395 & 034 & 190255.8 & +315940.9 & 0.635 & 1863 & 0.8 & 11.8 & FSRQ \\
\hline S4 2021+614 & 063 & 202206.5 & +613656.8 & 0.227 & 2623 & 1.4 & 14.0 & FSRQ \\
\hline PKS 2209+236 & 050 & 221205.9 & +235538.9 & 1.125 & 1123 & 1.7 & 6.5 & FSRQ \\
\hline 1Jy 2227-088 & 024 & 222939.9 & -083253.1 & 1.561 & 2423 & 2.2 & 4.5 & FSRQ \\
\hline 1Jy 2255-282 & 012 & 225805.8 & -275818.1 & 0.926 & 2127 & 9.8 & 2.2 & FSRQ \\
\hline 1Jy $2333-528$ & 195 & 233612.0 & -523620.8 & - & 1588 & $0.8^{*}$ & 1.7 & FSRQ \\
\hline 1Jy $2351+456$ & 074 & 235421.5 & +455303.1 & 1.992 & 1127 & 1.7 & 10.5 & FSRQ \\
\hline PKS 2355-534 & 189 & 235753.2 & -531112.8 & 1.006 & 1782 & $1.3^{*}$ & 2.0 & FSRQ \\
\hline
\end{tabular}

* $33 \mathrm{GHz}$ flux.

assuming a spectral slope $\alpha_{\mathrm{r}}=0.4\left(f \propto v^{-\alpha_{\mathrm{r}}}\right)$. All sources were detected above the 3 -sigma confidence level, with the exception of S4 $2021+614$, for which the X-ray fluxes is at the $\approx 2.1$-sigma confidence level.

The X-ray fluxes of Cols. 5 and 6 are fairly low, but typical of LBL blazars with similar radio fluxes. We verified this by comparing the distribution of the $\alpha_{\mu x}$ values listed in Col. 7 of Table 1 to the distribution of the same parameter in the sample of WMAP blazars detected both at $94 \mathrm{GHZ}$ and in the X-ray band considered by Giommi et al. (2005) (see Fig. 1). All values of $\alpha_{\mu x}$ in Table 2 are within the range observed in the sample of Giommi et al. (2006a), the two distributions (solid and dashed lines in Fig. 1) are similar, and the average value of $1.10 \pm 0.2$ is consistent with the average value (1.07) in the sample of Giommi et al. (2006a).

\subsection{Spectral analysis}

Spectral deconvolution can be performed only on those objects that were observed long enough to allow the detection of at least 130 photons in single or (when possible) merged observations. Table 3 list the subset of the XRT observations reported in Table 2 for which we have found more than 130 photons in single and/or merged observations; the dates for the considered observation are reported in Col. 2 of Table 3. The photon indices for the single and merged observations are reported in Col. 3 of this table and are labelled with the suffix $\left({ }^{a}\right)$ when the spectra are obtained from merged observations. Spectra were extracted from a circular region around the source with radius of 20 pixels, which includes $90 \%$ of the source photons. In order to use $\chi^{2}$ statistics, spectra were rebinned to include at least 20 photons in each energy channel. We used the XSPEC11.3 package and, given the very limited statistics, we only fitted the data to a simple power law spectrum with low energy absorption $\left(N_{\mathrm{H}}\right)$ fixed to the Galactic value in the direction of each source. A 5\% systematic error was included to take into account calibration uncertainties (Campana et al. 2006). The results are reported in Table 3 where Col. 1 gives the blazar name, Col. 2 gives the observation date, Col. 3 gives the best fit photon index with one sigma errors, Col. 4 the reduced $\chi^{2}$ and Col. 5 the number of degrees of freedom.

Best fit spectra were de-reddened using the cross sections of Morrison \& McCammon (1983) and included in the Spectral Energy Distributions (SEDs) shown in Figs. 2 through 4 that were built with non-simultaneous multi-frequency data taken from NED and other catalogs available at the ASDC.

For the objects for which we collected between 50 and 130 photons in each single observation listed in Table 2, a (power law) spectral slope was estimated from the hardness ratio between the $0.5-2.5 \mathrm{keV}$ and the $2.5-10 \mathrm{keV}$ band. In this case, no observation merging has been adopted in the spectral analysis. Again the amount of $N_{\mathrm{H}}$ was assumed to be equal to the Galactic value. The results are reported in Table 4 where Col. 1 gives the source name, Col. 2 the observation date, Cols. 3 and 4 the count-rates in the $0.5-2.5$ and $2.5-10.0 \mathrm{keV}$ bands, Col. 5 gives the (photon) spectral index derived from the hardness ratio. In all cases the spectral slopes are flat, consistent with the values obtained from the brighter sources, and indicative of inverse Compton emission.

\subsection{X-ray flux variability}

We searched for time variability both within single observations and in between separate exposures whenever a source was observed more than once. 
Table 2. XRT observations and results of X-ray image analysis on data taken in Photon Counting mode.

\begin{tabular}{|c|c|c|c|c|c|c|}
\hline Blazar name & Obs. date & $\begin{array}{c}\text { XRT exposure } \\
\text { seconds } \\
\text { (3) } \\
\end{array}$ & $\begin{array}{c}\text { XRT count rate } \\
0.3-10 . \mathrm{keV} \\
\text { cts/s } \\
(4) \\
\end{array}$ & $\begin{array}{c}\text { X-ray flux } \\
0.5-2 \mathrm{keV} \\
\mathrm{erg} \mathrm{cm}^{-2} \mathrm{~s}^{-1} \\
(5) \\
\end{array}$ & $\begin{array}{c}\text { X-ray flux } \\
2-10 \mathrm{keV}^{-2} \\
\mathrm{erg} \mathrm{cm}^{-2} \mathrm{~s}^{-1} \\
(6) \\
\end{array}$ & $\alpha_{\mu x}$ \\
\hline DW $0202+31$ & 24-Jun.-2006 & 11844 & $(2.48 \pm 0.16) \times 10^{-2}$ & $3.2 \times 10^{-13}$ & $9.3 \times 10^{-13}$ & 1.08 \\
\hline PKS 0215+015 & 28-Jun.-2005 & 9360 & $(6.0 \pm 0.30) \times 10^{-2}$ & $7.8 \times 10^{-13}$ & $2.1 \times 10^{-12}$ & 1.04 \\
\hline \multirow[t]{4}{*}{ PKS 0511-220 } & 30-Mar.-2006 & 2987 & $(1.36 \pm 0.23) \times 10^{-2}$ & $5.8 \times 10^{-13}$ & $1.5 \times 10^{-12}$ & 1.05 \\
\hline & 06-Apr.-2006 & 2781 & $(1.56 \pm 0.26) \times 10^{-2}$ & $2.2 \times 10^{-13}$ & $2.20 \times 10^{-13}$ & 1.12 \\
\hline & 10-Apr.-2006 & 4405 & $(9.6 \pm 1.6) \times 10^{-3}$ & $1.34 \times 10^{-13}$ & $1.34 \times 10^{-13}$ & 1.15 \\
\hline & 20-Apr.-2006 & 1029 & $(1.2 \pm 0.4) \times 10^{-2}$ & $1.68 \times 10^{-13}$ & $1.68 \times 10^{-13}$ & 1.13 \\
\hline S5 0633+73 & 09-Apr.-2006 & 11432 & $(2.07 \pm 0.15) \times 10^{-2}$ & $2.69 \times 10^{-13}$ & $7.87 \times 10^{-13}$ & 1.09 \\
\hline \multirow[t]{6}{*}{ 1Jy 0805-077 } & 22-May-2005 & 5847 & $(2.4 \pm 0.2) \times 10^{-2}$ & $3.1 \times 10^{-13}$ & $9.6 \times 10^{-13}$ & 1.09 \\
\hline & 26-May-2005 & 916 & $(2.5 \pm 0.6) \times 10^{-2}$ & $3.2 \times 10^{-13}$ & $1.0 \times 10^{-12}$ & 1.09 \\
\hline & 04-Jun.-2005 & 12955 & $(2.6 \pm 0.2) \times 10^{-2}$ & $3.3 \times 10^{-13}$ & $1.0 \times 10^{-12}$ & 1.09 \\
\hline & 11-Sep.-2005 & 4578 & $(2.3 \pm 0.3) \times 10^{-2}$ & $2.9 \times 10^{-13}$ & $9.2 \times 10^{-13}$ & 1.10 \\
\hline & 25-Sep.-2005 & 9016 & $(2.1 \pm 0.2) \times 10^{-2}$ & $2.7 \times 10^{-13}$ & $8.4 \times 10^{-13}$ & 1.10 \\
\hline & 01-Oct.-2005 & 3963 & $(1.9 \pm 0.3) \times 10^{-2}$ & $2.4 \times 10^{-13}$ & $7.6 \times 10^{-13}$ & 1.11 \\
\hline \multirow[t]{2}{*}{ 1Jy $1030+415$} & 21-Feb.-2006 & 3088 & $(2.17 \pm 0.30) \times 10^{-2}$ & $2.91 \times 10^{-13}$ & $6.38 \times 10^{-13}$ & 1.10 \\
\hline & 30-Jun.-2006 & 8746 & $(2.14 \pm 0.18) \times 10^{-2}$ & $2.87 \times 10^{-13}$ & $6.31 \times 10^{-13}$ & 1.10 \\
\hline \multirow[t]{3}{*}{ S5 1044+71 } & 16-Apr.-2005 & 1770 & $(4.3 \pm 0.5) \times 10^{-2}$ & $5.6 \times 10^{-13}$ & $1.5 \times 10^{-12}$ & 1.04 \\
\hline & 08-Jun.-2005 & 732 & $(3.9 \pm 0.8) \times 10^{-2}$ & $5.1 \times 10^{-13}$ & $1.4 \times 10^{-12}$ & 1.04 \\
\hline & 16-Nov.-2005 & 2159 & $(3.0 \pm 0.4) \times 10^{-2}$ & $3.9 \times 10^{-13}$ & $1.1 \times 10^{-13}$ & 1.06 \\
\hline PKS 1206-238 & 17-Dec.-2005 & 5744 & $(1.7 \pm 0.2) \times 10^{-2}$ & $2.2 \times 10^{-13}$ & $5.8 \times 10^{-13}$ & 1.10 \\
\hline 1 Jy $1213-172$ & 17-Dec.-2005 & 10282 & $(2.5 \pm 0.2) \times 10^{-2}$ & $3.3 \times 10^{-13}$ & $9.0 \times 10^{-13}$ & 1.10 \\
\hline \multirow[t]{2}{*}{ PKS 1313-333 } & 12-Jul.-2005 & 298 & $(5.5 \pm 1.5) \times 10^{-2}$ & $7.2 \times 10^{-13}$ & $2.0 \times 10^{-12}$ & 1.05 \\
\hline & 07-Sep.-2005 & 6358 & $(4.9 \pm 0.3) \times 10^{-2}$ & $6.4 \times 10^{-13}$ & $1.8 \times 10^{-12}$ & 1.06 \\
\hline 1Jy 1406-076 & 04-Sep.-2005 & 26457 & $(1.9 \pm 0.1) \times 10^{-2}$ & $2.5 \times 10^{-13}$ & $6.6 \times 10^{-13}$ & 1.11 \\
\hline \multirow[t]{2}{*}{ 1Jy $1424-418$} & 19-Apr.-2005 & 2269 & $(5.6 \pm 0.5) \times 10^{-2}$ & $7.2 \times 10^{-13}$ & $2.2 \times 10^{-12}$ & 1.08 \\
\hline & 23-Apr.-2005 & 1549 & $(3.3 \pm 0.5) \times 10^{-2}$ & $4.3 \times 10^{-13}$ & $1.3 \times 10^{-12}$ & 1.11 \\
\hline \multirow[t]{2}{*}{ 1Jy $1548+056$} & 15-Sep.-2005 & 5732 & $(4.1 \pm 0.3) \times 10^{-2}$ & $5.3 \times 10^{-13}$ & $1.5 \times 10^{-12}$ & 1.07 \\
\hline & 30-Sep.-2005 & 4445 & $(4.7 \pm 0.4) \times 10^{-2}$ & $6.1 \times 10^{-13}$ & $1.7 \times 10^{-12}$ & 1.0 \\
\hline \multirow[t]{2}{*}{ PKS 1725-795 } & 04-Feb.-2006 & 2721 & $(5.0 \pm 0.5) \times 10^{-2}$ & $6.5 \times 10^{-13}$ & $1.9 \times 10^{-12}$ & 1.02 \\
\hline & 20-Apr.-2006 & 3418 & $(6.3 \pm 0.5) \times 10^{-2}$ & $8.1 \times 10^{-13}$ & $2.4 \times 10^{-12}$ & 1.01 \\
\hline \multirow[t]{2}{*}{ S2 $1848+28$} & 17-May-2005 & 4546 & $(1.1 \pm 0.2) \times 10^{-2}$ & $1.4 \times 10^{-13}$ & $4.6 \times 10^{-13}$ & 1.11 \\
\hline & 18-Dec.-2005 & 5837 & $(1.9 \pm 0.2) \times 10^{-2}$ & $2.4 \times 10^{-13}$ & $7.9 \times 10^{-13}$ & 1.08 \\
\hline 3C 395 & 02-Apr.-2005 & 5015 & $(2.8 \pm 0.3) \times 10^{-2}$ & $3.5 \times 10^{-13}$ & $1.2 \times 10^{-12}$ & 1.05 \\
\hline S4 $2021+614$ & 06-Feb. 2006 & 2324 & $(2.7 \pm 1.3) \times 10^{-3}$ & $3.4 \times 10^{-14}$ & $1.2 \times 10^{-13}$ & 1.25 \\
\hline PKS 2209+236 & 27-Apr.-2005 & 1215 & $(1.1 \pm 0.3) \times 10^{-2}$ & $1.4 \times 10^{-13}$ & $4.1 \times 10^{-13}$ & 1.16 \\
\hline \multirow{2}{*}{ 1Jy $2227-088$} & 20-Apr.-2005 & 614 & $(2.0 \pm 0.2) \times 10^{-1}$ & $2.6 \times 10^{-12}$ & $7.2 \times 10^{-12}$ & 0.98 \\
\hline & 28-Apr.-2005 & 9249 & $(9.0 \pm 0.4) \times 10^{-2}$ & $1.2 \times 10^{-12}$ & $3.2 \times 10^{-12}$ & 1.03 \\
\hline 1Jy 2255-282 & 23-Apr.-2005 & 4313 & $(4.1 \pm 0.3) \times 10^{-2}$ & $5.3 \times 10^{-13}$ & $1.4 \times 10^{-12}$ & 1.19 \\
\hline 1Jy 2333-528 & 25-Nov.-2005 & 8991 & $(1.1 \pm 0.1) \times 10^{-2}$ & $1.4 \times 10^{-13}$ & $3.7 \times 10^{-13}$ & 1.11 \\
\hline \multirow[t]{2}{*}{$1 \mathrm{Jy} 2351+456$} & 17-May-2005 & 7930 & $(7.1 \pm 1.1) \times 10^{-3}$ & $9.1 \times 10^{-14}$ & $2.9 \times 10^{-13}$ & 1.19 \\
\hline & 17-Aug.-2005 & 1933 & $(1.3 \pm 0.3) \times 10^{-2}$ & $1.7 \times 10^{-13}$ & $5.3 \times 10^{-13}$ & 1.15 \\
\hline PKS 2355-534 & 17-Aug.-2005 & 4147 & $(8.4 \pm 0.5) \times 10^{-2}$ & $1.1 \times 10^{-12}$ & $2.9 \times 10^{-12}$ & 1.00 \\
\hline
\end{tabular}

No significant rapid variability (i.e. within single exposures) was detected in any source. Some marginal evidence for flux variability within a factor of $2.2 \pm 0.2$, and $1.8 \pm 0.3$ was only found between separate observations of the sources 1Jy 2227-088 and 1Jy 1424-418.

\section{Swift UVOT observations}

The Swift Ultraviolet and Optical Telescope (UVOT, Roming et al. 2005) is a $30 \mathrm{~cm}$ telescope equipped with two grisms and six broadband filters. UVOT is normally operated taking exposure with all filters during each Swift observation, unless a very bright $(\mathrm{mV}<12)$ object is present in the field of view.

The data of all WMAP sources that were sufficiently bright to be detected by UVOT in at least one filter were reduced with the SWIFTTOOLS software available within the standard HEASOFT software package.
Photometry was performed using a $6^{\prime \prime}$ aperture radius for the $V, B$ and $U$ filters and $12^{\prime \prime}$ radius for the $U V W 1, U V M 2$ and $U V W 2$ filters. The raw counts extracted in each aperture after correction for the coincidence loss (similar to photon pile-up in X-ray CCDs), were converted into standard magnitudes using the latest UVOT in-orbit zeropoint values (see e.g. Roming et al. 2005a, and reference therein for a full discussion about UVOT calibration procedures and photometric accuracy in each filter).

The magnitude were de-reddened using the maps of Schlegel et al. (1998) and the extinction curve of Cardelli et al. (1989) for the optical filters $(V, B$ and $U)$ and those of Seaton (1979) for the $U V$ filters ( $U V W 1, U V M 2$ and $U V W 2 A)$. The extinction corrected magnitudes were then converted to fluxes using the convertion factors included in the UVOT calibration data.

All useful data have been plotted as part of the SEDs shown in Figs. 2 through 4; the results of the UVOT observations of the brightest blazars are also reported in Table 5, where we report 


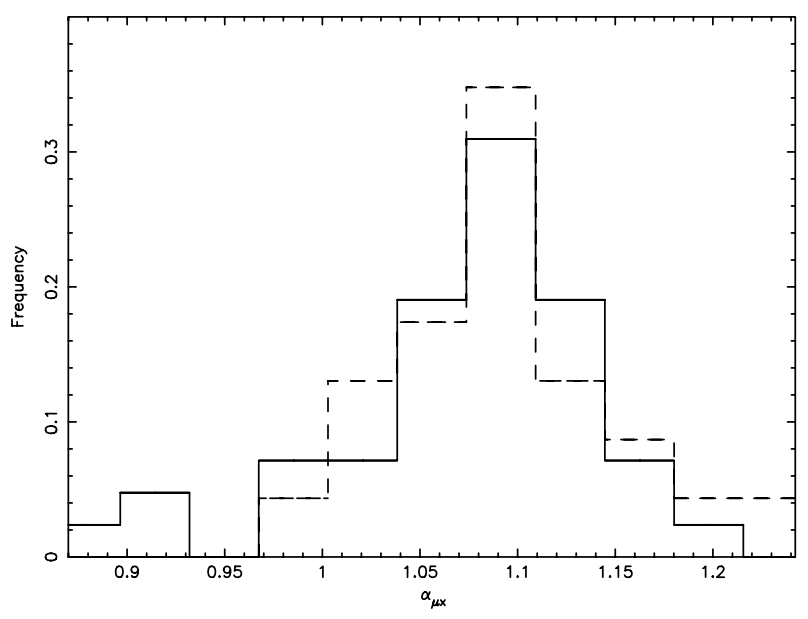

Fig. 1. The observed distributions of broad-band spectral slopes between the micro-wave ( $94 \mathrm{GHZ})$ and X-ray $(1 \mathrm{keV})$ bands of the sample of blazars detected in the $94 \mathrm{GHz}$ WMAP channel and for which X-ray data were available before our Swift observations (solid line, adapted from Giommi et al. 2006a) and of the sample presented here (dashed line).

Table 3. Results of XSPEC spectral analysis. Fits to simple power law model.

\begin{tabular}{ccccc}
\hline \hline Blazar name & Observation date & Photon index & $\chi_{\text {red }}^{2}$ & d.o.f. \\
\multicolumn{1}{c}{$(1)$} & $(2)$ & $(3)$ & $(4)$ & $(5)$ \\
\hline DW 0202+31 & 24-Jun.-2006 & $1.6 \pm 0.1$ & 1.26 & 11 \\
PKS 0215+015 & 28-Jun.-2005 & $1.53 \pm 0.07$ & 0.98 & 20 \\
PKS 0511-220 & 30-Mar.-2006 & $1.8 \pm 0.2^{a}$ & 0.4 & 4 \\
& 06-Apr.-2006 & & & \\
& 10-Apr.-2006 & & & \\
& 20-Apr.-2006 & & & \\
S5 0633+73 & 09-Apr.-2006 & $1.5 \pm 0$. & 0.54 & 8 \\
1Jy 0805-077 & 22-May-2005 & $1.2 \pm 0.2$ & 1.68 & 3 \\
& 04-Jun.-2005 & $1.6 \pm 0.1$ & 0.46 & 10 \\
& 25-Sep.-2005 & $1.6 \pm 0.2$ & 1.37 & 5 \\
1Jy 1030+415 & 21-Feb.-2006 & $1.6 \pm 0.1^{a}$ & 0.72 & 9 \\
& 30-Jun.-2006 & & & \\
1Jy 1213-172 & 17-Dec.-2005 & $1.8 \pm 0.1$ & 1.8 & 8 \\
PKS 1313-333 & 07-Sep.-2005 & $1.8 \pm 0.2$ & 1.03 & 9 \\
1Jy 1406-076 & 04-Sep.-2005 & $1.6 \pm 0.1$ & 0.79 & 17 \\
1Jy 1548+056 & 15-Sep.-2005 & $1.7 \pm 0.2$ & 1.93 & 6 \\
PKS 1725-795 & 04-Feb.-2006 & $1.6 \pm 0.1^{a}$ & 0.8 & 13 \\
& 20-Apr.-2006 & & & \\
3C 395* & 02-Apr.-2005 & $1.6 \pm 0.4$ & 1.53 & 4 \\
3C 395** & 02-Apr.-2005 & $1.7 \pm 0.2$ & 1.47 & 18 \\
1Jy 2227-088 & 28-Apr.-2005 & $1.54 \pm 0.08$ & 1.09 & 29 \\
1Jy 2255-282 & 23-Apr.-2005 & $1.7 \pm 0.3$ & 0.77 & 4 \\
PKS 2355-534 & 17-Aug.-2005 & $1.5 \pm 0.2$ & 0.53 & 4 \\
\hline AnS
\end{tabular}

${ }^{a}$ Analysis performed on data merged from all observations.

* Photon Counting data, ${ }^{* *}$ Windowed timing data (exposure of $6185 \mathrm{~s}$ ).

the Blazar name (Col. 1), the UVOT observation date (Col. 2), the UVOT filter (Col. 3) and the observed magnitude (Col. 4).

\section{Summary and discussion}

We have presented in this paper the results of 41 Swift observations of all the 23 micro-wave-selected blazars that were never reported as X-ray emitters in a $41 \mathrm{GHz}$ flux-limited sample of 140 blazars detected as bright WMAP foreground sources.
The main motivations that prompted this study are: $i$ ) establish the X-ray properties of a statistically representative sample of micro-wave selected blazars that are known to contaminate in a non-negligible way CMB fluctuation maps; ii) verify or exclude the existence of a population of X-ray quiet blazars; iii) compare the X-ray properties of these sources with those of blazars selected in different energy bands; and iv) complete the X-ray measurements of a micro-wave flux-limited sample of blazars that is useful for multi-frequency statistical studies.

We found that all blazars were clearly detected by the Swift X-Ray Telescope even in very short exposures. The observed $2-10 \mathrm{keV}$ fluxes range between $2.2 \times 10^{-13}$ and $5.1 \times$ $10^{-12} \mathrm{erg} \mathrm{cm}^{-2} \mathrm{~s}^{-1}$ and are well within the expectations for LBL type blazars with radio flux densities close to $1 \mathrm{Jy}$. We therefore conclude that there is no evidence for a population of $\mathrm{X}$-ray quiet blazars.

The X-ray spectral slopes of the objects for which spectral analysis was possible are flat, typical of inverse Compton emission as can be seen from Tables 3 and 4 and from the SEDs shown in Figs. 2, through 4. In fact, all the SEDs shown in Figs. 2 through 4 clearly show that all our objects are of the LBL type with the Swift XRT X-ray data sampling the rise of the Inverse Compton emission, while the UVOT data represent either the tail of the synchrotron component or, in some cases, the UV blue bump.

We have also looked for time variability in our data since flux variations of the inverse Compton component of blazars is not well known, as most of the blazars that have been monitored at these frequencies are of the HBL type. We found that no rapid variability was detected in any source. For the sake of completeness, we mention that fairly large X-ray flux variations have been, however, observed in the inverse Compton emission from e.g. 3C 273 and 3C 279 (Turner et al. 1990; Ballo et al. 2002; Giommi et al. 2002; Courvoisier et al. 2003) but only in exposures separated by months or years. Large variations (of a factor of a few) on time scales of days, were also recently detected in the LBL blazar 3C 454.3 by the Swift XRT in a series of observations carried out during a giant optical and X-ray flare (Giommi et al. 2006b; Pian et al. 2006).

Since the detection of all the 23 objects considered in this work brings the detection rate in the X-ray band to $100 \%$ of the 140 WMAP confirmed blazars with $41 \mathrm{GHz}$ flux larger than $0.8 \mathrm{Jy}$, we can safely conclude that all micro-wave selected blazars are $X$-ray sources with radio to X-ray flux ratio similar to that of blazars selected at $\mathrm{GHz}$ frequencies, and in general of LBL blazars.

The micro-wave to X-ray spectral slopes $\left(\alpha_{\mu x}\right.$, see Eq. (1)) of our sources are all within the very tight range seen in the sample of WMAP sources considered by Giommi et al. (2006a). We therefore confirm that the $\alpha_{\mu x}$ distribution of all WMAP blazars of the LBL type ( $\approx 85 \%$ of the entire population) is very narrow and, consequently, that the X-ray flux is a very good estimator of the micro-wave flux.

This result may also apply to radio galaxies as the average $\alpha_{\mu x}$ of the 6 objects of this type included both in the WMAP catalog and in the Rosat all sky survey is 1.04 , very similar to that of blazars $\left(\left\langle\alpha_{\mu x}\right\rangle=1.07\right)$. We note, however, that the soft X-ray emission in radio galaxies is not necessarily dominated by inverse Compton radiation as it may include other components like, e.g., radiation from an accretion disk, etc. The $\alpha_{\mu x}$ distribution of radio galaxies could therefore have a lower average and, probably, a wider dispersion.

As shown by Giommi \& Colafrancesco (2004) and by Giommi et al. (2006a), blazars are the main foreground 
Table 4. Results of hardness-ratio spectral analysis assuming a simple power law model.

\begin{tabular}{|c|c|c|c|c|}
\hline $\begin{array}{c}\text { Blazar name } \\
\text { (1) } \\
\end{array}$ & $\begin{array}{c}\text { Observation date } \\
\text { (2) } \\
\end{array}$ & $\begin{array}{c}\text { Count rate } \\
0.5-2.5 \mathrm{keV} \\
(3) \\
\end{array}$ & $\begin{array}{c}\text { Count rate } \\
2.5-10 \mathrm{keV} \\
(4) \\
\end{array}$ & $\begin{array}{c}\text { Photon index } \\
\text { (5) } \\
\end{array}$ \\
\hline S5 1044+71 & 16-Apr.-2005 & $(2.9 \pm 1.4) \times 10^{-2}$ & $(1.0 \pm 0.3) \times 10^{-2}$ & $1.6 \pm 0.4$ \\
\hline PKS 1206-238 & 17-Dec.-2005 & $(1.2 \pm 0.2) \times 10^{-2}$ & $(3.7 \pm 0.9) \times 10^{-3}$ & $1.7 \pm 0.2$ \\
\hline \multirow{2}{*}{ 1Jy $1424-418$} & 19-Apr.-2005 & $(3.7 \pm 0.4) \times 10^{-2}$ & $(1.9 \pm 0.3) \times 10^{-2}$ & $1.4 \pm 0.2$ \\
\hline & 23-Apr.-2005 & $(1.9 \pm 0.4) \times 10^{-2}$ & $(1.2 \pm 0.3) \times 10^{-2}$ & $1.2 \pm 0.3$ \\
\hline \multirow[t]{2}{*}{ S2 $1848+28$} & 17-May-2005 & $(7.9 \pm 1.4) \times 10^{-3}$ & $(2.5 \pm 0.8) \times 10^{-3}$ & $1.8 \pm 0.3$ \\
\hline & 18-Dec.-2005 & $(1.3 \pm 0.2) \times 10^{-2}$ & $(5.7 \pm 1.2) \times 10^{-3}$ & $1.5 \pm 0.2$ \\
\hline 1Jy $2333-528$ & 25-Nov.-2005 & $(7.4 \pm 1.0) \times 10^{-3}$ & $(2.8 \pm 0.6) \times 10^{-3}$ & $1.5 \pm 0.2$ \\
\hline $1 \mathrm{Jy} 2351+456$ & 17-May-2005 & $(4.5 \pm 0.8) \times 10^{-3}$ & $(2.5 \pm 0.6) \times 10^{-3}$ & $1.4 \pm 0.2$ \\
\hline
\end{tabular}
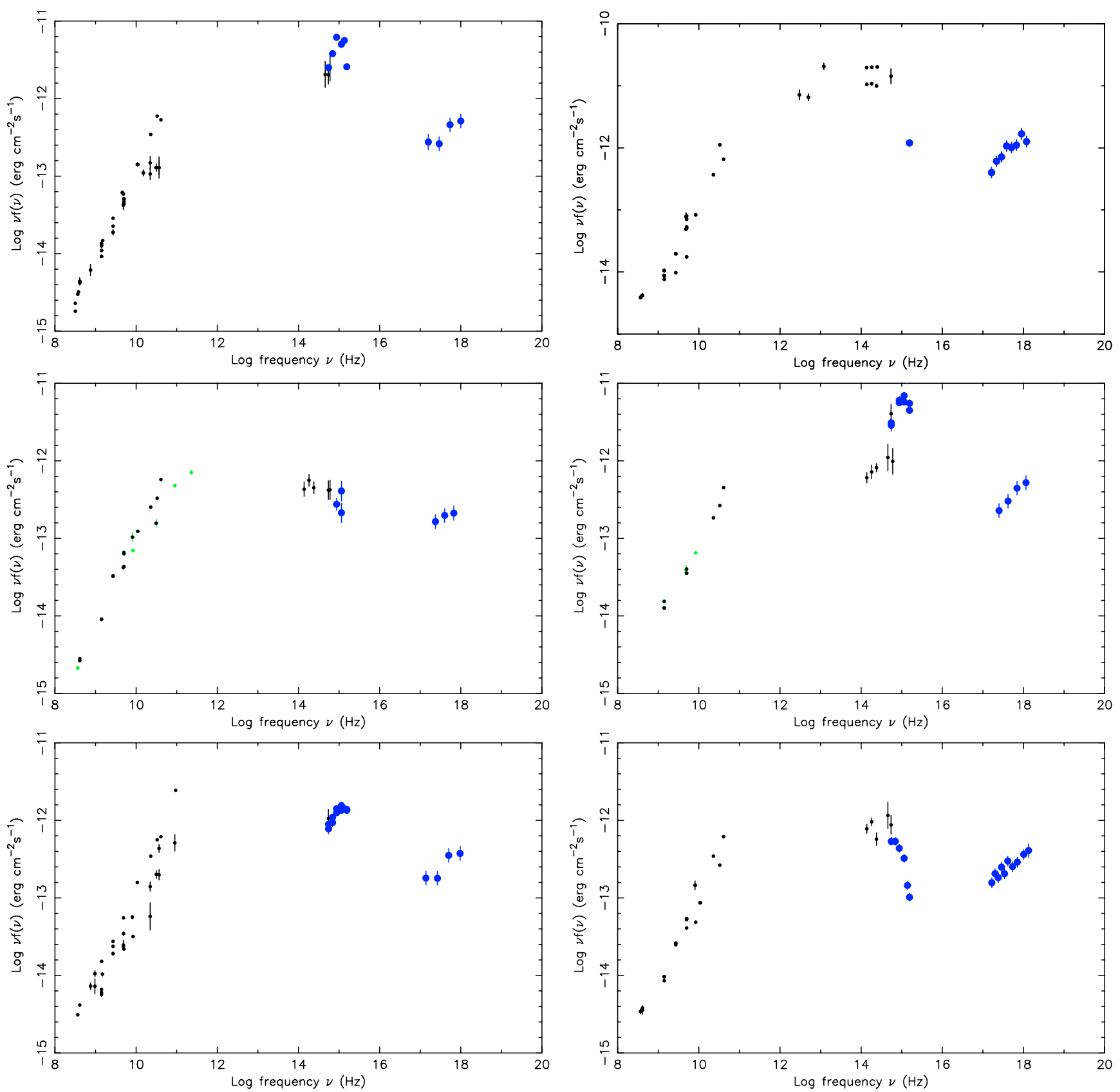

Fig. 2. Top left. The Spectral Energy Distribution of the blazar DW 0202+31 = WMAP085. Top right. The Spectral Energy Distribution of the blazar PKS 0215+015 = WMAP096. Mid left. The Spectral Energy Distribution of the blazar PKS 0511-220= WMAP127. Mid right. The Spectral Energy Distribution of the blazar S5 0633+73 = WMAP087. Bottom left. The Spectral Energy Distribution of the blazar 1Jy 1030+415 = WMAP103. Bottom right. The Spectral Energy Distribution of the blazar 1Jy 1406-07 = WMAP203. Swift-XRT and UVOT data are shown as large filled circles. 

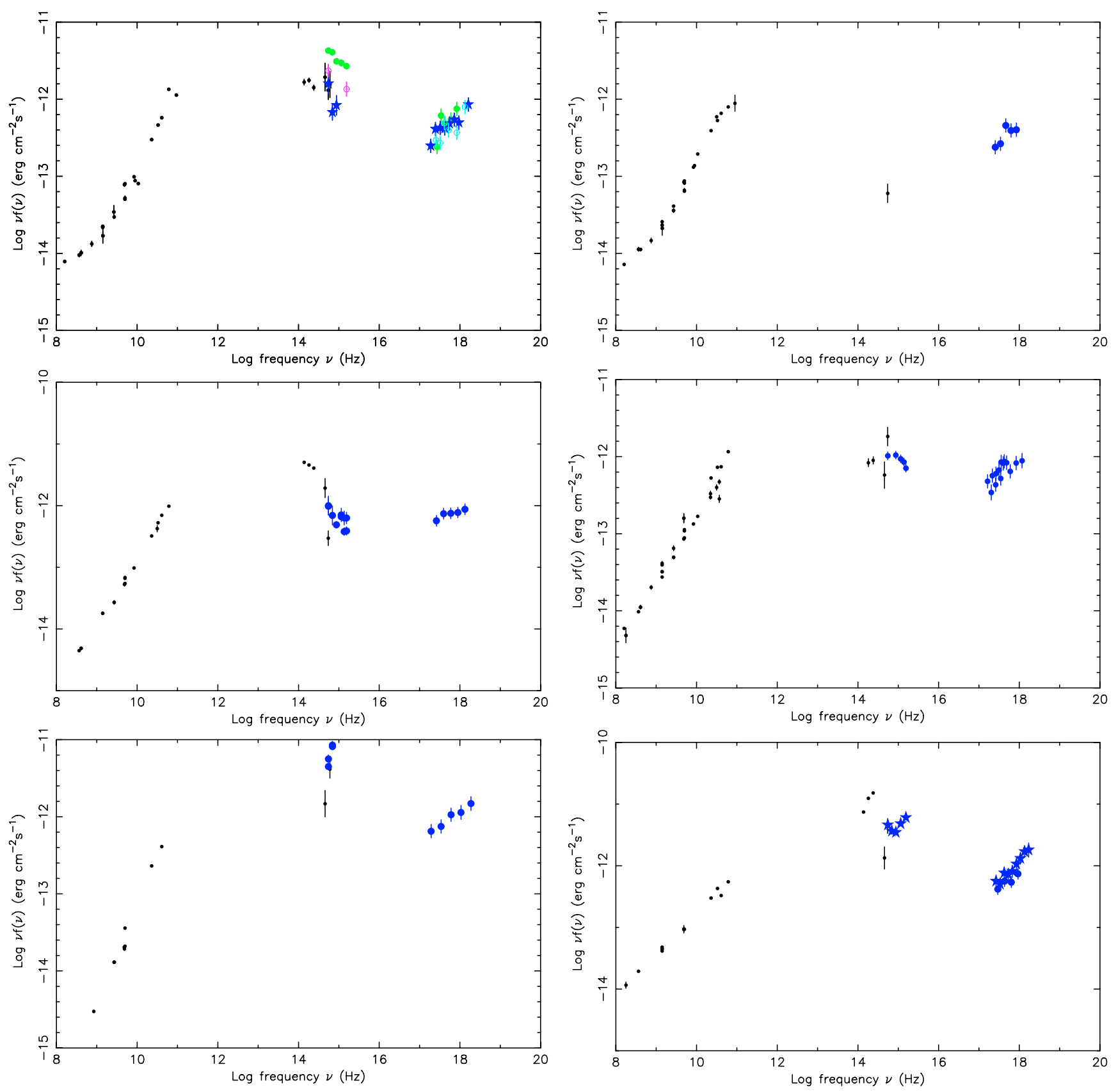

Fig. 3. Top left. The Spectral Energy Distribution of the blazars 1Jy 0805-077 = WMAP133. Swift-XRT are shown as filled stars (22 May 2005), open circles (4 June 2005) and filled circles (25 September 2005). UVOT data were taken only on May 22 (filled stars), June 4 (open circles) and October 1 (filled circles). Note that while the optical/UV data (end of the synchrotron component) shows significant variability, the X-ray data (inverse Compton component) are consistent with a constant flux. Top right. The Spectral Energy Distribution of the blazar $1 \mathrm{Jy} 1213-172=$ WMAP173. Swift-XRT data taken on 17 December 2005 are shown as large filled circles. No UVOT data are available for this source because of the presence of a 2.8 mag star (HD106625) in the UVOT field of view. Mid left. The Spectral Energy Distribution of the blazar PKS 1313-333 = WMAP182. Swift-XRT and UVOT data taken on 7 September 2005 are shown as large filled circles. Mid right. The Spectral Energy Distribution of the blazar 1Jy 1548+056 = WMAP007. Swift-XRT and UVOT data are shown as large filled circles. Bottom left. The Spectral Energy Distribution of the blazar PKS 1725-795 = WMAP186. Swift-XRT and UVOT data are shown as large filled circles. Bottom right. The Spectral Energy Distribution of the blazar 3C $395=$ WMAP034. Swift-XRT (Windowed timing) and UVOT data are shown as large star symbols and large filled circles (Photon Counting data).

contaminants of CMB anisotropy maps and can alter the reconstruction of the CMB fluctuation spectrum in a significant way. The measurement of the X-ray flux of blazars and radio galaxies over a large area of the sky would therefore be extremely useful to locate a large number of these sources and to determine their clustering properties. Giommi et al. (2006a) showed that a deep all sky survey with flux limit of the order of $10^{-15} \mathrm{erg} \mathrm{cm}^{-2} \mathrm{~s}^{-1}$ in the soft X-ray band would detect well over 100000 blazars, a database that could be used to perform a careful cleaning of high sensitivity CMB data from Planck and future CMB missions. This data set would also be ideally suited for a detailed estimate of the spatial correlation function of blazars and radio 

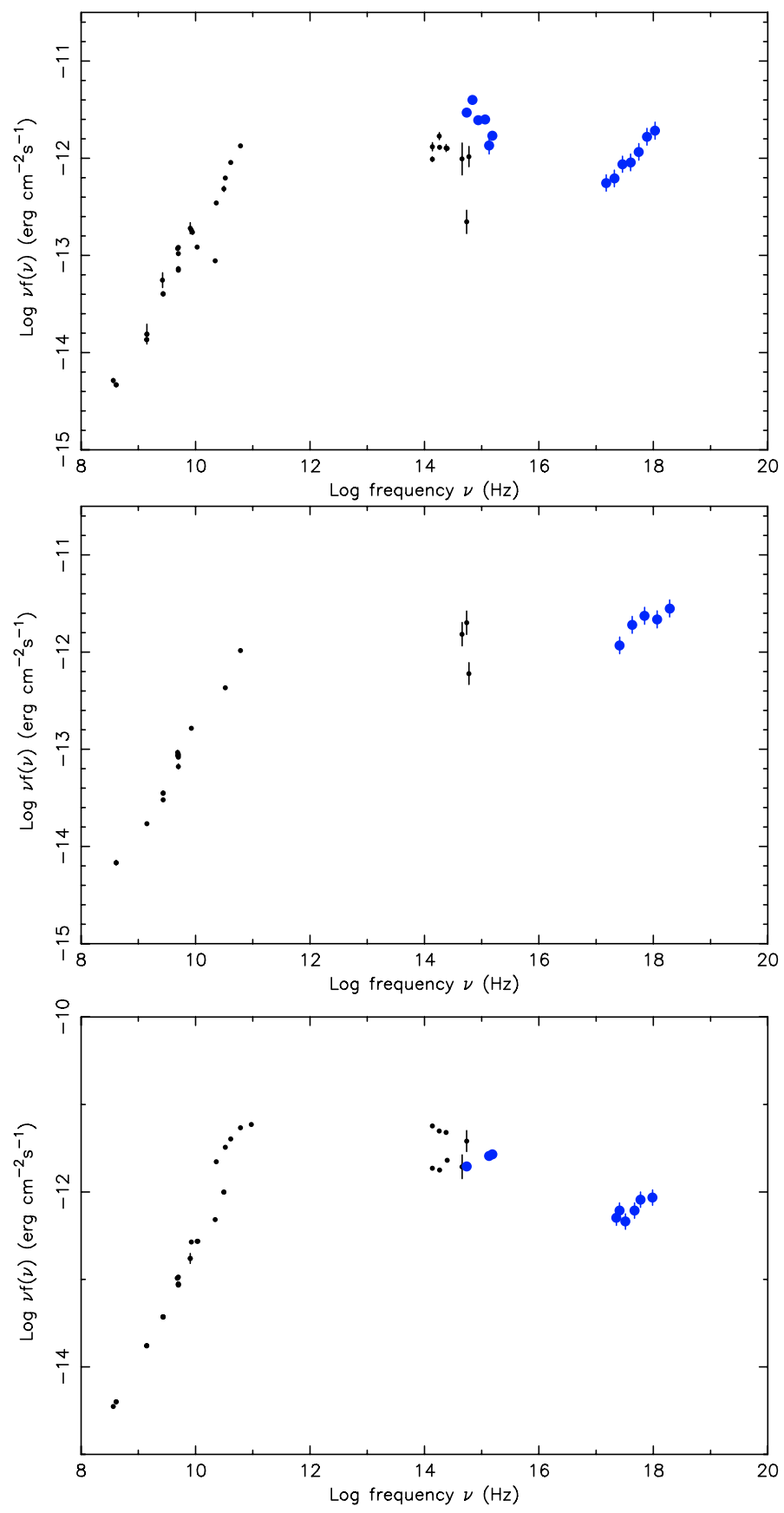

Fig. 4. Top. The Spectral Energy Distribution of the blazar 1Jy 2227$088=$ WMAP024. Swift-XRT and UVOT data are shown as large filled circles. Mid. The Spectral Energy Distribution of PKS 2355-534 = WMAP189. Swift-XRT and UVOT data are shown as large filled circles. Bottom. The Spectral Energy Distribution of 1Jy 2255-282 = WMAP012. Swift-XRT and UVOT data are shown as large filled circles.

galaxies; this information could be used to infer the residual impact of even fainter objects of this type on the CMB temperature and polarization fluctuation spectrum.

The observations presented here provide valuable optical/UV and X-ray data for most WMAP blazars that lacked this type of broad-band multi-frequency information. The WMAP micro-wave flux-limited sample of blazars complements the few existing and statistically well defined samples selected at other frequencies like e.g. the 1Jy BL Lac sample
Table 5. Results of UVOT observations.

\begin{tabular}{cccc}
\hline \hline Blazar name & Observation date & Filter & mag \\
& & & \\
(1) & $(2)$ & $(3)$ & $(4)$ \\
\hline DW 0202+31 & 24-Jun.-2006 & W1 & 17.16 \\
& & M2 & 17.77 \\
& & W2 & 18.53 \\
PKS 0215+015 & 28-Jun.-2005 & W2 & 17.73 \\
PKS 0511-220 & 06-Apr.-2006 & W1 & 20.22 \\
S5 0633+73 & 09-Apr.-2006 & W1 & 18.09 \\
& & W2 & 19.28 \\
1Jy 0805-077 & 22-May-2005 & W1 & 16.42 \\
& & M2 & 16.07 \\
& & W2 & 18.87 \\
1Jy 1030+415 & 21-Feb.-2006 & W1 & 17.40 \\
& & W2 & 17.98 \\
PKS 1206-238 & 17-Dec.-2005 & W1 & 18.50 \\
& & M2 & 19.28 \\
& & W2 & 18.98 \\
PKS 1313-333 & 07-Sep.-2005 & W1 & 17.97 \\
& & M2 & 18.24 \\
& & W2 & 18.43 \\
PKS 1725-795 & 04-Feb.-2006 & W1 & 17.06 \\
& & W2 & 17.44 \\
1Jy 2227-088 & 28-Apr.-2005 & W1 & 16.94 \\
& & M2 & 17.92 \\
& & W2 & 17.76 \\
1Jy 2255-282 & 23-Apr.-2005 & M2 & 16.72 \\
& & W2 & 16.86 \\
1Jy 2351+456 & 17-May-2005 & W1 & 17.67 \\
& & M2 & 18.49 \\
& & W2 & 18.41 \\
\hline
\end{tabular}

(Stickel et al. 1991), the EMSS (Rector et al. 2000), the Einstein Slew survey (Elvis et al. 1992; Perlman et al. 1996) etc. Complete/flux limited samples with extensive multi-frequency data are very important for the investigation of blazar statistical properties from different viewpoints: a particularly interesting and relatively new one is the $\gamma$-ray band that is about to be deeply explored by the upcoming AGILE and GLAST missions.

Acknowledgements. We acknowledge financial support by the Italian Space Agency (ASI) through grant I/R/039/04 and through funding of the ASI Science Data Center. The UCL-MSSL authors acknowledge support of PPARC. We wish to thank all members of the Swift team who made this very flexible mission possible. This work is partly based and on data taken from the NASA/IPAC Extragalactic Database (NED) and from the ASI Science Data Center (ASDC).

\section{References}

Ballo, L., Maraschi, L., Tavecchio, F., et al. 2002, ApJ, 567, 50 Barthelmy, S. D., Barbier, L. M., Cummings, J. R., et al. 2005, SSRv., 120, 95 Burrows, D. N., Hill, J. E., Nousek, J. A., et al. 2005, SSRv., 120, 165 Bennett, C. L., Bay, M., Halpern, M., et al. 2003a, ApJ, 583, 1

Bennett, C. L., Hill, R. S., Hinshaw, G., et al. 2003b, ApJS, 148, 97 Blandford, R. D., \& Rees, M. J. 1978, in Pittsburgh Conference on BL Lac Objects, ed. A. M. Wolfe (University of Pittsburgh, Pittsburgh), 328

Campana, S., Beardmore, A. P., Cusumano, G., \& Godet, O. 2006, http://swift.gsfc.nasa.gov/docs/heasarc/caldb/swift/docs/ xrt/SWIFT-XRT-CALDB-09.ps

Cardelli, J. A., Clayton, G. C., \& Mathis, J. S. 1989, ApJ, 345, 245

Collinge, M. J., Strauss, M. A., Hall, P. B., et al. 2005, AJ, in press

Courvoisier, T. J.-L., Beckmann, V., Bourban, G., et al. 2003, A\&A, 411, 343

Dickey, J. M., \& Lockman, F. J. 1990, ARA\&A, 28, 215 
Elvis, M., Plummer, D., Schachter, J., \& Fabbiano, G. 1992, ApJS, 80, 257 Gehrels, N., Chincarini, G., Giommi, P., et al. 2004, ApJ, 611, 1005 Giommi, P., \& Colafrancesco, S. 2004, A\&A, 414, 7

Giommi, P., Massaro, E., Chiappetti, L., et al. 1999, A\&A, 351, 59

Giommi, P., Capalbi, M., Fiocchi, M., et al. 2002, in Blazar Astrophysics with BeppoSAX and Other Observatories, ed. P. Giommi, E. Massaro, \& G. Palumbo, 63

Giommi, P., Piranomonte, S., Perri, M., \& Padovani, P. 2005, A\&A, 434, 385

Giommi, P., Colafrancesco, S., Cavazzuti, E., et al. 2006a, A\&A, 445, 843

Giommi, P., Blustin, A., Capalbi, M., et al. 2006b, A\&A, 456, 911

Hartman, R. C., Bertsch, D. L., Bloom, S. D., et al. 1999, ApJS, 123, 79

Hill, J. E., et al. 2004, SPIE, 5165, 217

Morrison, R., \& McCammon, D. 1983, ApJ, 270, 119

Padovani, P., \& Giommi, P. 1995, ApJ, 444, 567

Padovani, P., Perlman, E., Landt, H., Giommi, P., \& Perri, M. 2003, ApJ, 58, 128
Perlman, E. S., Stocke, J. T., Schachter, J. F., et al. 1996, ApJS, 104, 251

Pian, E., Foschini, A., Beckmann, V., et al. 2006, A\&A, 449, L21

Ravasio, M., Tagliaferri, G., Ghisellini, G., et al. 2002, A\&A, 383, 763

Rector, T. A., Stocke, J. T., Perlman, E. S., Morris, S. L., \& Gioia, I. M. 2000, AJ, 120, 1626

Roming, P. W. A., Kennedy, T. E., Mason, K. O., et al. 2005, SSRv, 120, 143

Schlegel, D. J., Finkbeiner, D. P., \& Davis, M. 1998, ApJ, 500, 525

Seaton, M. J. 1979, MNRAS, 187, 73

Stickel, M., Fried, J. W., Kühr, H., Padovani, P., \& Urry, C. M. 1991, ApJ, 374, 431

Tagliaferri, G., Ghisellini, G., Giommi, P. et al. 2000, A\&A, 354, 431

Turner, M. J. L., Williams, O. R., Courvoisier, et al. 1990, MNRAS, 244, 310

Urry, C. M., \& Padovani, P. 1995, PASP, 107, 803

Voges, W., Aschenbach, B., Boller, T., et al. 1999, A\&A, 349, 389 